\title{
Decomposições matriciais para escoamentos viscoelásticos incompressíveis
}

\author{
Irineu L. Palhares Junior, Cassio M. Oishi, \\ Depto de Matemática e Computação, FCT, UNESP, \\ 19060-900, Presidente Prudente, SP \\ E-mail: irineulopespalhares@gmail.com, cassiooishi@gmail.com,
}

\begin{abstract}
Resumo: Uma dificuldade na solução de escoamentos viscoelásticos complexos ocorre quando instabilidades numéricas surgem na simulação, resultantes de um colapso ("breakdown") dos esquemas numéricos aplicados na solução da equação constitutiva para fluidos não-newtonianos. Essa dificuldade é conhecida na literatura como o Problema de Alto Número de Weissenberg ou "High Weissenberg Number Problem"(HWNP). Neste trabalho, investigamos decomposições matriciais aplicadas ao tensor conformação $\boldsymbol{A}$ empregues como métodos de estabilização na simulação do HWNP. Após isso, no contexto do método "Marker-and-Cell", empregamos discretizações por diferenças finitas juntamente com o método de projeção na implementação das decomposições matriciais, visando solucionar o HWNP. O código é verificado utilizando o problema de um escoamento bidimensional entre placas paralelas ("Poiseuille flow") para um fluido Oldroyd-B. Finalmente, realizamos um estudo comparativo entre os métodos de estabilização em termos de precisão, estabilidade e eficiência computacional.
\end{abstract}

Palavras-chave: Instabilidades numéricas, Problema de Alto Número de Weissenberg, Escoamentos viscoelásticos, Diferenças finitas

\section{Introdução}

O estudo de escoamentos de fluidos viscoelásticos tem grande destaque em reologia computacional, uma vez que em aplicações industriais muitos materiais comportam-se como fluidos viscoelásticos. Assim, o estudo destes escoamentos tem despertado o interesse de muitos pesquisadores. Entretanto, apesar do crescente avanço na área, problemas de instabilidade numérica vem surgindo juntamente com esse progresso. Em especial, uma dificuldade conhecida como Problema de Alto Número de Weissenberg ou "High Weissenberg Number Problem" (HWNP). Este é um fenômeno numérico que acarreta em instabilidades e/ou na não convergência da solução, mesmo com diferentes formulações ou formas de discretização (diferenças finitas, volumes finitos, elementos finitos, entre outras).

Desta forma, diversos métodos de estabilização foram desenvolvidos para combater o HWNP. Em especial, destacamos os métodos que fazem uso de decomposições matriciais empregues ao tensor conformação A que é simétrico e definido positivo. Assim, estes métodos de estabilização visam garantir a preservação desta propriedade, cuja degradação está associada com os efeitos do HWNP.

Assim, neste trabalho propomo-nos investigar alguns destes métodos de estabilização, baseados na decomposição do tensor conformação $\mathbf{A}$, a saber os métodos log-conformação desenvolvido por Fattal e Kupferman [3, 4], decomposição do tipo raiz quadrada apresentado por Balci et al. [2] e núcleo-conformação proposto por Afonso et al. [1]. 


\section{Equações básicas}

Escoamentos de fluidos viscoelásticos incompressíveis podem ser descritos matematicamente pelas equações de Navier-Stokes, equações (1) e (2), que representam, respectivamente, a lei de conservação da quantidade de movimento e conservação de massa, acrescidas da equação constitutiva do tensor conformação A, equação (3). Assim, as equações de Navier-Stokes juntamente com a equação constitutiva do tensor conformação, são dadas, respectivamente, por

$$
\begin{gathered}
\frac{\partial \mathbf{u}}{\partial t}+\nabla \cdot(\mathbf{u u})=-\nabla p+\frac{\beta}{R e} \nabla^{2} \mathbf{u}+\nabla \cdot \boldsymbol{\tau}, \\
\nabla \cdot \mathbf{u}=0, \\
\frac{\partial \mathbf{A}}{\partial t}+\nabla \cdot(\mathbf{u A})=\nabla \mathbf{u} \mathbf{A}+\mathbf{A} \nabla \mathbf{u}^{T}+\frac{1}{W i} f(\mathbf{A}) P(\mathbf{A}),
\end{gathered}
$$

onde $\mathbf{u}$ é o campo de velocidade, $p$ a pressão, $\beta$ é a razão entre a viscosidade do solvente $\nu_{s}$ e a viscosidade total $\nu_{o}$ (onde $\nu_{o}=\nu_{s}+\nu_{p}$ ), Re $=\frac{H U \rho}{\nu_{o}}$ é o número de Reynolds (onde $\rho$ é a densidade, $U$ e $H$ são parâmetros que determinam as escalas de velocidade e comprimento, respectivamente), $W i=\lambda \frac{U}{H}$ o número de Weissenberg (onde $\lambda$ é o tempo de relaxação), $\boldsymbol{\tau}$ o tensor polimérico e $\mathbf{A}$ o tensor conformação. As funções $f$ e $P$ que aparecem na equação (3) determinam o tipo de fluido viscoelástico (Oldroyd-B, Giesekus, FENE-CR e FENE-P), sendo $f$ uma função escalar e $P$ um polinômio de $\mathbf{A}$.

As condições de contorno adotadas dividem-se em três grupos: região de entrada de fluido, paredes sólidas e região de saída de fluido. Na região de entrada de fluido atribuímos as soluções analíticas da velocidade e da contribuição não-newtoniana. Para a região de saída de fluido, aplicamos a condição de Neumann homogêneo. Por fim, nas paredes sólidas empregamos a condição de não escorregamento e impermeabilidade para a velocidade, de modo que as condições, nesta região, para a contribuição não-newtoniana são deduzidas pela simplificação da equação (3).

\section{Decomposições matriciais}

\subsection{Log-conformação}

Nesta subseção, apresentamos a formulação log-conformação, proposta por Fattal e Kupferman $[3,4]$, para a equação constitutiva (3). Este método tornou-se uma das principais técnicas de suporte às instabilidades numéricas causadas pelo HWNP. Em suma, este método reformula a equação constitutiva do tensor conformação mediante duas etapas principais, uma decomposição aplicada ao tensor $\nabla \mathbf{u}$ e, posteriormente, aplicamos uma transformação logarítmica em A, construindo uma nova equação constitutiva para a variável logarítmica concebida.

A seguir, apresentamos o teorema que descreve a decomposição do gradiente da velocidade $\nabla \mathbf{u}$, empregue na equação (3), que viabiliza a construção de uma equação evolutiva para a variável logarítmica do tensor conformação $\mathbf{A}$.

Teorema 1. Seja u um campo de velocidade e $\boldsymbol{A}$ o tensor conformação que é simétrico definido positivo. Então, o gradiente da velocidade $\nabla \boldsymbol{u}$ pode ser decomposto como:

$$
\nabla \boldsymbol{u}=\boldsymbol{\Omega}+\boldsymbol{B}+\boldsymbol{N} \boldsymbol{A}^{-1},
$$

onde $\boldsymbol{\Omega}=\boldsymbol{\Omega}(\nabla \boldsymbol{u}, \boldsymbol{A})$ e $\boldsymbol{N}=\boldsymbol{N}(\nabla \boldsymbol{u}, \boldsymbol{A})$ são antissimétricas, e $\boldsymbol{B}=\boldsymbol{B}(\nabla \boldsymbol{u}, \boldsymbol{A})$ é simétrica $e$ comuta com o tensor conformação $\boldsymbol{A}$. 
Mediante a substituição de (4) na equação constitutiva para o tensor conformação (3), obtemos uma nova equação constitutiva para o tensor conformação $\mathbf{A}$, dada por

$$
\frac{\partial \mathbf{A}}{\partial t}+\nabla \cdot(\mathbf{u A})=(\boldsymbol{\Omega} \mathbf{A}-\mathbf{A} \boldsymbol{\Omega})+2 \mathbf{B} \mathbf{A}+\frac{f(\mathbf{A})}{W i} P(\mathbf{A}) .
$$

O tensor conformação $\mathbf{A}$, assim como qualquer matriz simétrica, pode ser decomposto como

$$
\mathbf{A}=\mathbf{O} \mathbf{\Lambda} \mathbf{O}^{T}
$$

onde $\mathbf{O}$ é a matriz ortogonal dos autovetores e $\boldsymbol{\Lambda}$ é a matriz diagonal dos autovalores de $\mathbf{A}$.

Desta maneira, definimos o logaritmo do tensor conformação como

$$
\boldsymbol{\Psi}=\ln (\mathbf{A})=\mathbf{O} \ln (\mathbf{\Lambda}) \mathbf{O}^{T},
$$

que implica, pela propriedade inversa, em

$$
e^{\Psi}=\mathbf{A}
$$

Por conseguinte, substituindo (8) na equação (5), e aplicando algumas operações algébricas, obtemos a seguinte equação de evolução para $\Psi$ :

$$
\frac{\partial \boldsymbol{\Psi}}{\partial t}+\nabla \cdot(\mathbf{u} \boldsymbol{\Psi})=(\boldsymbol{\Omega} \boldsymbol{\Psi}-\boldsymbol{\Psi} \boldsymbol{\Omega})+2 \mathbf{B}+\frac{f\left(e^{\boldsymbol{\Psi}}\right)}{W i} e^{-\boldsymbol{\Psi}} P\left(e^{\boldsymbol{\Psi}}\right) .
$$

\subsection{Decomposição do tipo raiz quadrada}

Há uma grande dificuldade em se construir esquemas numéricos que sejam vantajosos em termos de precisão e estabilidade simultaneamente. Em vista disso, Balci et al. [2] propuseram uma nova formulação para a equação constitutiva (3), baseada na matriz raiz quadrada do tensor conformação, que, segundo os autores, resultou em benefícios tanto em termos de precisão como, também, estabilidade. Além disso, é de fácil implementação e não há aumento significativo no custo computacional, que é equiparável à evolução direta do tensor conformação $\mathbf{A}$ (ou do tensor polimérico $\boldsymbol{\tau}$ ), uma vez que este método não requer o cálculo dos autovalores e autovetores do tensor conformação.

O tensor conformação A possui uma única decomposição da forma

$$
\mathbf{A}=\mathbf{Q}^{2}
$$

onde $\mathbf{Q}$ é a matriz raiz quadrada de $\mathbf{A}$, que também é uma matriz simétrica definida positiva.

Assim, na busca de uma equação evolutiva para $\mathbf{Q}$ substituímos (10) em (3), juntamente com algumas manipulações algébricas, que resulta em

$$
\frac{\partial \mathbf{Q}}{\partial t}+\nabla \cdot(\mathbf{u} \mathbf{Q})=\mathbf{G} \mathbf{Q}+\mathbf{Q} \nabla \mathbf{u}^{T}+\frac{f\left(\mathbf{Q}^{2}\right)}{2 W i} \mathbf{Q}^{-1} P\left(\mathbf{Q}^{2}\right),
$$

onde $\mathbf{G}$ é uma matriz antissimétrica.

\subsection{Núcleo-conformação}

Em vista do demasiado entusiasmo e bons resultados alcançados com o emprego de modificações na equação constitutiva do tensor conformação, uma questão que despertou o interesse dos estudiosos Afonso et al. [1] foi a possibilidade de reunir as principais sugestões de cada uma das decomposições em uma única técnica, na qual por meio de uma simples modificação na função principal, denominada função núcleo "kernel", escolhemos qual das estratégias adotar. Assim, na busca por tal método, Afonso et al. [1] desenvolveram o método núcleo-conformação, que, em particular, reuni os métodos log-conformação $[3,4]$ e decomposição do tipo raiz quadrada [2]. 
Esta formulação vai além do que simplesmente englobar algumas das técnicas já desenvolvidas, uma vez que cria a possibilidade do uso de outras transformações não investigadas até então, como a função inversa de seno hiperbólico, função logarítmica em qualquer base positiva, função raiz k-ésima, entre outras.

Definimos a função núcleo $\mathbb{K}()$ como

$$
\mathbb{K}(\mathbf{A})=\mathbf{O} \mathbb{K}(\boldsymbol{\Lambda}) \mathbf{O}^{T}
$$

onde $\mathbb{K}$ é uma função que deve satisfazer três condições: ser contínua, inversível e diferenciável. A construção desta equação se dá por meio de três etapas principais: construção de uma equação evolutiva para $\boldsymbol{\Lambda}$, uma equação evolutiva para $\mathbb{K}(\boldsymbol{\Lambda})$ e, por fim, uma equação de evolução para $\mathbb{K}(\mathbf{A})$. Desta forma, a equação de evolução para $\mathbb{K}(\mathbf{A})$ é dada por

$$
\frac{D \mathbb{K}(\mathbf{A})}{D t}=\boldsymbol{\Omega} \mathbb{K}(\mathbf{A})-\mathbb{K}(\mathbf{A}) \boldsymbol{\Omega}+2 \mathbb{B}+\frac{1}{W i} \mathbb{H},
$$

onde $\mathbb{B}=\mathbf{O} \tilde{\mathbf{B}} \mathbf{\Lambda} \mathbf{J} \mathbf{O}^{T}\left(\operatorname{com} \tilde{\mathbf{B}}=\mathbf{O}^{T} \mathbf{B O}\right.$ e $\left.\mathbf{J}=\frac{\partial \mathbf{J}}{\partial \boldsymbol{\Lambda}}\right)$ e $\mathbb{H}=\mathbf{O} f(\boldsymbol{\Lambda}) P(\boldsymbol{\Lambda}) \mathbf{J O}^{T}$.

\section{Escoamento entre placas paralelas}

Para as simulações consideramos um escoamento em um canal de dimensões $5 H \times H$, onde $H$ é a largura do canal e $5 H$ o seu comprimento. Em todas as simulações tomamos $H=1$. A verificação será feita através da comparação entre a solução adquirida com o emprego das decomposições matriciais e a solução analítica do problema. Mais precisamente, apresentaremos os erros relativos e o cálculo da taxa de convergência espacial de cada uma das técnicas. Além dos métodos de estabilização, também apresentamos os resultados com o método padrão, desprovido de decomposições. O modelo constitutivo adotado em todas as simulações será o modelo Oldroyd-B. Ademais, realizaremos um estudo preliminar da eficiência computacional, precisão e estabilidade dos métodos.

A partir de agora, denotaremos os métodos padrão, log-conformação, decomposição do tipo raiz quadrada, núcleo-conformação aplicado às transformações linear $\mathbb{K}(\mathbf{A})=\xi(\mathbf{A}-\mathbf{I})$ e raiz quadrada $\mathbb{K}(\mathbf{A})=\mathbf{A}^{\frac{1}{2}}$, por Standard, Ln, Square-root, Kernel-linear e Kernel-root, respectivamente.

Os testes foram executados em duas malhas diferentes $M_{1}(\delta h=0.05,20 \times 100)$ e $M_{2}$ $(\delta h=0.025,40 \times 200)$, onde $\delta h$ é o passo espacial. O passo temporal é definido como $\delta t=0.001$ em todas as simulações realizadas. Os parâmetros básicos usados nas simulações são $R e=0.01$ e $\beta=0.5$. Os valores do número de Weissenberg utilizados nos testes são $W i=0.1,1,2$ e 3, obtidos mediante a variação do tempo de relaxação $\lambda$. Os resultados apanhados foram selecionados fazendo-se um corte vertical no centro do canal, ou seja, fixamos $x=2.5 \mathrm{H}$, e variamos o valor de $y$.

\subsection{Precisão}

O estudo de precisão é realizado mediante a análise dos erros relativos dos métodos. O cálculo dos erros relativos foram realizados pela norma $L_{2}$. A Tabela 1 , para $W i=1$, apresenta o valor do erro relativo e taxa de convergência espacial dos métodos de estabilização para a velocidade $u$ e a componente $\tau_{11}$ do tensor polimérico $\tau$. Todos os métodos apresentaram exatamente os mesmos bons resultados. Além disso, note que os erros diminuíram com o refinamento de malha, de $M_{1}$ para $M_{2}$, demonstrando convergência dos resultados. Isto pode ser verificado pela taxa de convergência espacial para todos os métodos, na qual temos apenas valores positivos. Enquanto a taxa de convergência espacial para a velocidade é próximo a 2, para a componente $\tau_{11}$ do tensor polimérico este valor é reduzido, sendo um pouco maior que 1. Além disso, na Figura 1 apresentamos o perfil das componentes $\tau_{11}$ e $\tau_{12}$ do tensor polimérico $\boldsymbol{\tau}$ com os métodos de 
estabilização e da solução analítica. Observamos que a solução com o emprego dos métodos apresentam um comportamento muito próximo da solução analítica.

\begin{tabular}{ccccccc}
\hline Oldroyd-B & $E\left(u^{M_{1}}\right)$ & $E\left(u^{M_{2}}\right)$ & $O(u)$ & $E\left(\tau_{11}^{M_{1}}\right)$ & $E\left(\tau_{11}^{M_{2}}\right)$ & $O\left(\tau_{11}\right)$ \\
\hline Standard & $1.52 \times 10^{-3}$ & $3.86 \times 10^{-4}$ & 1.97 & $1.33 \times 10^{-2}$ & $5.45 \times 10^{-3}$ & 1.28 \\
Ln & $1.52 \times 10^{-3}$ & $3.87 \times 10^{-4}$ & 1.97 & $1.33 \times 10^{-2}$ & $5.45 \times 10^{-3}$ & 1.28 \\
Square-root & $1.52 \times 10^{-3}$ & $3.86 \times 10^{-4}$ & 1.97 & $1.33 \times 10^{-2}$ & $5.45 \times 10^{-3}$ & 1.28 \\
Kernel-linear & $1.52 \times 10^{-3}$ & $3.87 \times 10^{-4}$ & 1.97 & $1.33 \times 10^{-2}$ & $5.45 \times 10^{-3}$ & 1.28 \\
Kernel-root & $1.52 \times 10^{-3}$ & $3.86 \times 10^{-4}$ & 1.97 & $1.33 \times 10^{-2}$ & $5.45 \times 10^{-3}$ & 1.28 \\
\hline
\end{tabular}

Tabela 1: Erros e taxa de convergência espacial para a componente $u$ da velocidade e a componente $\tau_{11}$ do tensor polimérico no tempo $t=50$ e $W i=1$.

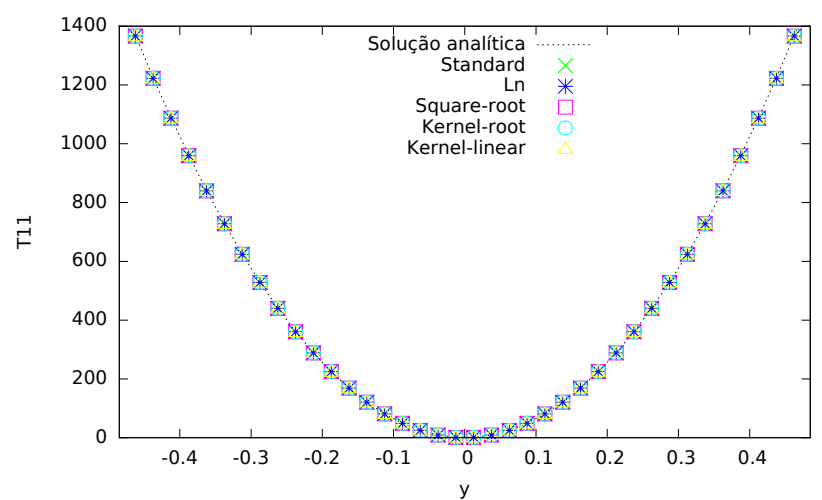

(a)

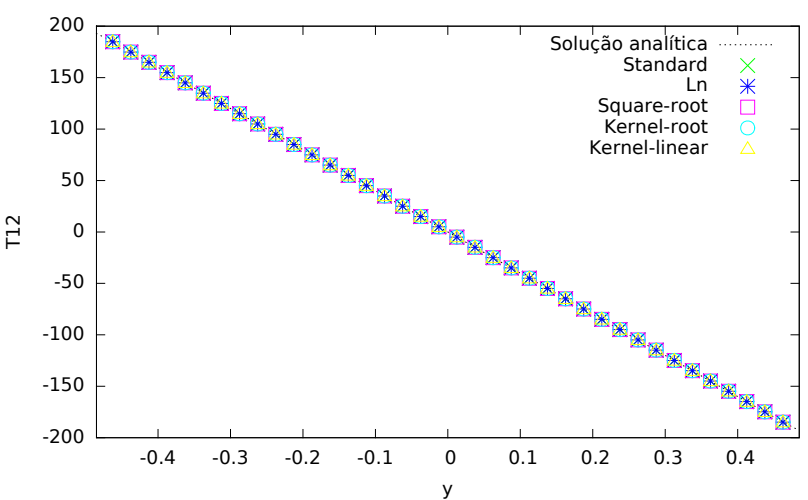

(b)

Figura 1: Simulação do escoamento entre placas paralelas com o modelo Oldroyd-B para $W i=1$, $t=50$ e malha $M_{2}$ : (a) perfil da componente $\tau_{11}(0.5, y)$ (b) perfil da componente $\tau_{12}(0.5, y)$

A Tabela 2 apresenta os erros relativos e a taxa de convergência espacial das variáveis $u$ e $\tau_{11}$ para $W i=2$. Observamos que este aumento no número de Weissenberg acarretou em erros distintos entre os métodos, diferente do que ocorreu para $W i=1$. Em especial, destacamos os erros obtidos com os métodos Square-root e Kernel-root que demonstraram um aumento significativo. Sendo mais perceptível para o método Kernel-root com ambas as componentes, $u$ e $\tau_{11}$, e para o método Square-root com a componente $\tau_{11}$. Além disso, o refinamento de malha não diminuiu os erros com o método Kernel-root. Esse fenômeno é representado pelo valor negativo da taxa de convergência de $\tau_{11}$ e $\tau_{12}$, indicando não haver convergência. Em contrapartida, os métodos Standard, Ln e Kernel-linear, continuam a apresentar bons resultados. A Figura 2 atesta as observações mencionadas para este valor de $W i$. Observamos que o perfil das componentes $\tau_{11}$ e $\tau_{12}$ do tensor polimérico $\boldsymbol{\tau}$ apresentam uma pequena deformação próximo aos contornos para os métodos Square-root e Kernel-root. Por outro lado, os métodos Standard, Ln e Kernel-linear apresentaram valores muito próximos da solução analítica.

\begin{tabular}{ccccccc}
\hline Oldroyd-B & $E\left(u^{M_{1}}\right)$ & $E\left(u^{M_{2}}\right)$ & $O(u)$ & $E\left(\tau_{11}^{M_{1}}\right)$ & $E\left(\tau_{11}^{M_{2}}\right)$ & $O\left(\tau_{11}\right)$ \\
\hline Standard & $1.19 \times 10^{-3}$ & $2.87 \times 10^{-4}$ & 2.05 & $1.08 \times 10^{-2}$ & $4.89 \times 10^{-3}$ & 1.14 \\
Ln & $1.22 \times 10^{-3}$ & $2.87 \times 10^{-4}$ & 2.08 & $1.09 \times 10^{-2}$ & $5.01 \times 10^{-3}$ & 1.12 \\
Square-root & $1.48 \times 10^{-3}$ & $3.44 \times 10^{-4}$ & 2.10 & $2.96 \times 10^{-1}$ & $7.62 \times 10^{-2}$ & 1.95 \\
Kernel-linear & $1.19 \times 10^{-3}$ & $2.87 \times 10^{-4}$ & 2.05 & $1.08 \times 10^{-2}$ & $4.89 \times 10^{-3}$ & 1.14 \\
Kernel-root & $1.22 \times 10^{-3}$ & $1.10 \times 10^{-2}$ & -4.11 & $1.08 \times 10^{-2}$ & $1.18 \times 10^{-1}$ & -4.68 \\
\hline
\end{tabular}

Tabela 2: Simulação do escoamento entre placas paralelas com o modelo Oldroyd-B para $W i=2$, $t=50$ e malha $M_{2}$ : (a) perfil da componente $\tau_{11}(0.5, y)$ (b) perfil da componente $\tau_{12}(0.5, y)$ 


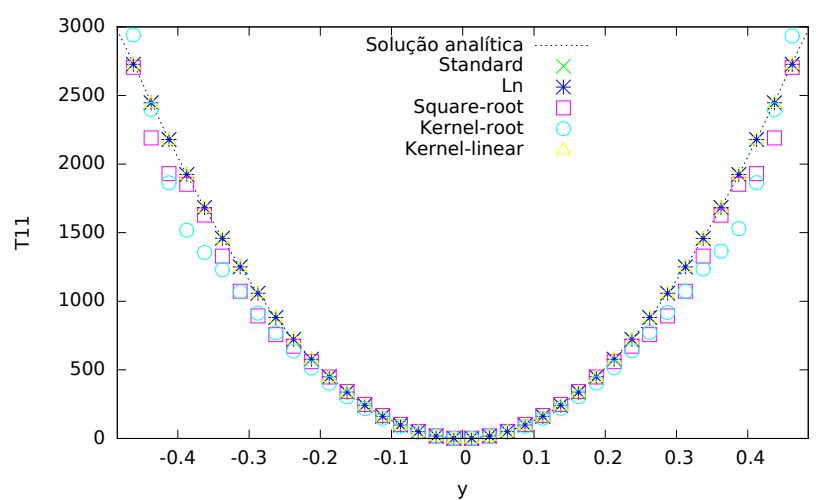

(a)

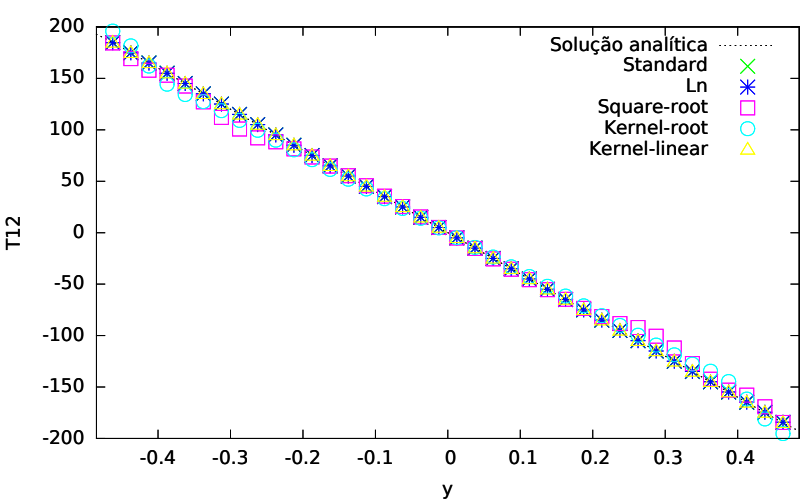

(b)

Figura 2: Simulação do escoamento entre placas paralelas com o modelo Oldroyd-B para $W i=2$, $t=50$ e malha $M_{2}$ : (a) perfil da componente $\tau_{11}(0.5, y)$ (b) perfil da componente $\tau_{12}(0.5, y)$

\subsection{Estabilidade}

Outro aspecto fundamental deste estudo diz respeito à estabilidade dos métodos. Faremos esta análise com base na capacidade do método em preservar a positividade do tensor conformação $\mathbf{A}$, que será averiguada mediante um monitoramento, em todo o domínio espacial, do determinante mínimo $\left(\operatorname{det}_{\min }(\mathbf{A})\right)$ do tensor conformação, a cada passo de tempo. A Tabela 3 apresenta o valor do determinante mínimo do tensor conformação $\mathbf{A}$ para todos os métodos de estabilização considerados, além do método Standard, para diferentes valores de $W i, W i=0.1,1,2$ e 3 , no tempo $t=50$. Observamos na Tabela 3 que o primeiro método a perder a positividade de $\mathbf{A}$ é o método Square-root. Além disso, diferentemente dos demais métodos, o $\operatorname{det}_{\min }(\mathbf{A})$ deste método, juntamente com $W i=2$, explode negativamente. Este fato deve-se a um crescimento ilimitado dos termos convectivos em (11), comprometendo toda a solução. Os métodos Standard e Kernel-linear, embora apresentem um valor positivo para o $\operatorname{det}_{\min }(\mathbf{A})$ em $t=50$, perdem a positividade para $W i=3$, uma vez que há a presença de valores negativos em tempos anteriores. Não observamos a perda da positividade de $\mathbf{A}$, até $W i=3$, para os métodos Ln e Kernel-root.

\begin{tabular}{cccccc}
\hline$W i$ & Standard & Ln & Square-root & Kernel-linear & Kernel-root \\
\hline 0.1 & 1.00 & 1.00 & 1.00 & 1.00 & 1.00 \\
1 & 1.00 & 1.00 & 1.00 & 1.00 & 1.00 \\
2 & 1.04 & 1.04 & $-3.80 \times 10^{40}$ & 1.04 & 1.03 \\
3 & $1.00 \times 10^{3}$ & 1.08 & $4.52 \times 10^{-4}$ & $1.00 \times 10^{3}$ & 1.08 \\
\hline
\end{tabular}

Tabela 3: Valor do $\operatorname{det}_{\text {min }}(\mathbf{A})$ para $W i=0.1,1,2$ e 3 no tempo $t=50$ com os métodos Standard, Ln, Square-root, Kernel-linear e Kernel-root. A malha usada foi $M_{2}$.

\subsection{Eficiência}

Finalmente, faremos uma breve análise em termos de eficiência computacional. Este estudo é realizado mediante a comparação da quantidade de tempo despendido para a simulação de cada um dos métodos, até atingir o tempo adimensional $t=50$. Todas as simulações foram executadas em uma máquina $1 \times$ Intel Core $i 7$ de 3.47 GHz com 24 GB RAM. Para a execução desta análise, realizamos apenas um teste para $W i=1$.

A Tabela 4 exibe o tempo gasto na simulação de cada um dos métodos para $W i=1 \mathrm{com}$ as malhas $M_{1}$ e $M_{2}$. Como sugerido pela construção dos métodos, a técnica mais eficiente, dentre os métodos com o uso de decomposições matriciais, é o método Square-root. Isto pode ser observado na Tabela 4, na qual, independentemente das malhas adotadas, o método em questão possui praticamente o mesmo custo computacional que o método Standard. Já os métodos Ln e Kernel- 
root, com o maior custo computacional, apresentam tempos de CPU semelhantes, uma vez que estes dispõem de uma estrutura muito similar, mudando apenas a transformação empregada. $\mathrm{O}$ método Kernel-linear está numa posição intermediária. Não é tão eficiente quanto os métodos Standard e Square-root, mas também, não apresenta um custo computacional tão alto como os métodos Ln e Kernel-root. Possivelmente, esta redução no custo do método Kernel-linear, deve-se à sua transformação mais simples, quando comparado à transformação logarítmica e raiz quadrada.

\begin{tabular}{ccc}
\hline Método & $M_{1}$ & $M_{2}$ \\
\hline Standard & $04: 39$ & $25: 09$ \\
Ln & $07: 57$ & $37: 40$ \\
Square-root & $04: 48$ & $25: 48$ \\
Kernel-linear & $06: 12$ & $31: 09$ \\
Kernel-root & $07: 59$ & $37: 50$ \\
\hline
\end{tabular}

Tabela 4: Tempo de CPU (min: seg) para $W i=1$ e $t=50$.

De modo geral, não podemos apontar com certeza qual o método estabilizador com o melhor desempenho, uma vez que, cada método estabilizador teve um desempenho melhor em um determinado quesito. Além disso, salientamos que as observações postas anteriormente é exclusivamente para o problema do canal, com as configurações adotadas neste trabalho. Portanto, não podemos assegurar estas mesmas características para outros problemas, parâmetros e modelos constitutivos.

\section{Considerações finais}

As informações expostas neste trabalho servem como um amparo no estudo dos assuntos pertinentes às instabilidades do HWNP e de algumas das principais propostas de estabilização desenvolvidas envolvendo decomposições matriciais do tensor conformação. Além disso, esta análise serve como um auxílio para uma escolha mais acertada de um método estabilizador, levando-se em conta os parâmetros, a geometria e o modelo constitutivo adotados neste trabalho.

\section{Referências}

[1] A. M. Afonso, F. T. Pinho, M. A. Alves, The kernel-conformation constitutive laws, J. Non-Newtonian Fluid Mech., 167-168 (2012) 30-37.

[2] N. Balci , B. Thomases, M. Renardy, C. R. Doering, Symmetric factorization of the conformation tensor in viscoelastic fluid models, J. Non-Newtonian Fluid Mech., 166 (2011) 546-553.

[3] R. Fattal, R. Kupferman, Constitutive laws for the matrix-logarithm of the conformation tensor, J. Non-Newtonian Fluid Mech., 123 (2004) 23-37.

[4] R. Fattal, R. Kupferman, Time-dependent of viscoelastic flows at high Weissenberg number using the log-conformation representation, J. Non-Newtonian Fluid Mech., 126 (2005) 2337. 